\title{
The micro-Resistive WELL detector for the phase-2 upgrade of the LHCb muon detector
}

\section{Poli Lener*}

Laboratori Nazionali di Frascati - INFN, Frascati, Italy

E-mail: marco.polilenerelnf.infn.it

\section{G. Bencivenni, G. Felici, M. Gatta, G. Morello, E. Tskahadadze}

Laboratori Nazionali di Frascati - INFN, Italy

\section{R. De Oliveira}

CERN, $\mathrm{CH}$

\section{A. Ochi}

University of Kobe, Japan

\begin{abstract}
The $\mu$-RWELL is a new generation Micro-Pattern Gaseous Detector, composed of two elements: the cathode and the $\mu$-RWELL_PCB including the amplification stage, realized with a polyimide structure micro-patterned with a blind-hole matrix, embedded through a Diamond-Like Carbon (DLC) resistive layer with the readout PCB. Different layouts of the resistive stage have been studied: the simplest one is based on a single DLC layer with edge grounding, suitable for low rate applications $\left(30-40 \mathrm{kHz} / \mathrm{cm}^{2}\right)$. More sophisticated schemes are under study for high rate purposes (up to 2-3 MHz/cm ${ }^{2}$ ). The high-rate versions are still under intense R\&D in order to optimize the performance and the constructive process but this detector seems already a valid candidate to be used for the phase 2 upgrade of the LHCb muon detector. For this upgrade, the experiment is targeting a luminosity of $2 \times 10^{34} \mathrm{~cm}^{-2} \mathrm{~s}^{-1}$, with strong requirements on the robustness and detection efficiency of the muon system. We report on the ongoing R\&D, showing also the latest measured performances of the new high rate versions of the detector.
\end{abstract}

The 39th International Conference on High Energy Physics (ICHEP2018)

4-11 July, 2018

Seoul, Korea

${ }^{*}$ Speaker. 


\section{Introduction}

The LHCb experiment $[1,2]$ is a single-arm spectrometer dedicated to the study of the CP violation and other rare phenomena in the decay of Beauty particles. One of its feature is a fast and versatile trigger system to select the interesting events. The apparatus is designed like a fixedtarget experiment due to the very forward peaked b-quark distribution at LHC. It is composed of five systems: vertex, tracking, ring Cerenkov detectors, the calorimeters and the muon system. Up to the end of $2017 \mathrm{LHCb}$ has recorded a total luminosity of $7 \mathrm{fb}^{-1}$ and in the next year, since LHC is going to increase its luminosity, the apparatus needs to upgrade its system. For the first phase only the replacement of the FEE will be done. For the phase 2, the detectors should show a rate capability up to $3 \mathrm{MHz} / \mathrm{cm}^{2}$, an efficiency for single gap $>95 \%$ within $25 \mathrm{~ns}$ (BX), stability up to $6 \mathrm{C} / \mathrm{cm}^{2}$ integrated charge in $10 \mathrm{y}$ at $\mathrm{G}=4000$. So we propose for this upgrade the micro-Resistive WELL.

\section{The $\mu$-Resistive WELL}

The $\mu$-RWELL has been invented at LNF [3] with the aim to simplify the detector assembly, avoiding time consuming operations (i.e. epoxy curing cycle) and to improve the operation stability under heavy irradiation. The detector looks very compact and merges the features of two well-

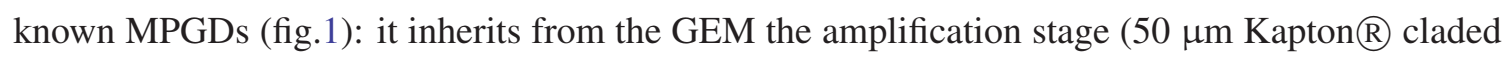
on one side with $5 \mu \mathrm{m} \mathrm{Cu}$ layer patterned with high density of holes) and from the MM the presence of a high resistive (10-200 M $\Omega / \square$ ) layer (DLC- diamond like carbon) above the readout plane. This layer mitigates the passage to the streamer region due to the drop of the amplification field strongly quenching the amplitude of the sparks. The surface resistivity must be optimized since features like the rate capability, the response speed and the spatial resolution are strongly dependent on the chosen application (HEP, neutron or gamma detection).

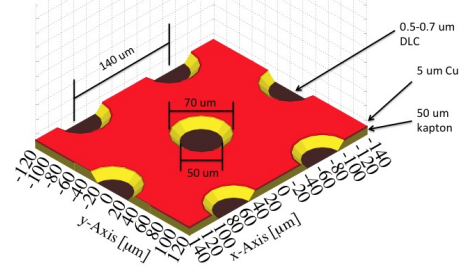

Figure 1: Sketch of a ирти-RWELL with the geometrical parameters.

\section{The $\mu$-RWELL and the TT}

The realization of the $\mu$-RWELL_PCB has been assigned to industry, with the aim to reduce the cost of the device. So far the very fruitful collaboration with the ELTOS S.p.A in San Zeno (AR, Italy) allowed the production of several $\mu$-RWELL_PCB with $10 \times 10 \mathrm{~cm}^{2}$ active area as well as the production of large prototypes (about $0.5 \mathrm{~m}^{2}$ ) for a proposal for CMS. The DLC sputtering 
has been instead done at Be-Sputter ltd sited in Japan, while the chemical opening of the amplification channels (blind holes) is done at the PCB-Workshop of CERN (by one of the authors). A collaboration is ongoing also with TECHTRA z.o.o. (POLAND).

\section{Different layouts for different applications}

The industrialization process requires a simpler layout, with a single resistive layer; such layout shows limitations strictly related to the rate [4], due to the same process leading to the charge amplitude quenching. In order to realize a product that can find room for an industrial production, with the purpose to reduce the costs, and that can stand up to high rate particles $\left(O\left(\mathrm{MHz} / \mathrm{cm}^{2}\right)\right.$ different charge evacuation schemes have been proposed, realized and tested, all with a single resistive layer: Silver Grid v1 (SGv1), Silver Grid v2 (SGv2) and Resistive Grid (RG) which features are reported in table 1.

\begin{tabular}{|c|c|c|c|c|}
\hline HR scheme & n. layers & pitch & type & Dead area \\
\hline \hline SG v1 & single & $6 \mathrm{~mm}$ & cond. grid & $33 \%$ \\
\hline SG v2 & single & $12 \mathrm{~mm}$ & cond. grid & $10 \%$ \\
\hline RG & single & $6 \mathrm{~mm}$ & resist. grid & - \\
\hline DL & double & $6+6 \mathrm{~mm}$ & cond. vias & - \\
\hline
\end{tabular}

Table 1: Summary of the features of the new single-resistive high rate versions of the $\mu$-RWELL together with the Double Layer (DL), the first high rate-oriented $\mu$-RWELL realized.

\section{Detector performances}

The mentioned detectors have been irradiated with X-rays at LNF. The detectors have been operated at a gain of 6300 and flushed with $\mathrm{Ar}: \mathrm{CO}_{2}: \mathrm{CF}_{4}$ 45:15:40. The rate capability over two orders of magnitude of estimated photon conversion have been measured and it is reported in fig. 2. The SGv1 can stand up to $3 \mathrm{MHz} / \mathrm{cm}^{2}$ losing the $10 \%$ of the gain. But, as reported in table 1 , this detector exhibits the largest dead area, so studies are ongoing to merge this rate capability with a smaller dead area. The detectors have been moreover exposed to the $\mathrm{H} 8 \mathrm{C}$ pion/muon beam in order to evaluate the tracking efficiency and they have been equipped with an analog FEE: APV25 readout by the SRS system [6]. As shown in fig. 3 the RG and the DL prototypes reach full efficiency ( $\sim 98 \%$ - No dead area in the amplification stage), while the SG 1 and SG2 show lower efficiency $(\sim 74 \%$ and $\sim 92 \%$ respectively), but higher than their geometrical acceptance (66\% and $90 \%$ respectively), thanks to the efficient electron collection mechanism of the combines drift/amplification electric field, reducing the effective dead zone. A measurement of the time resolution has been performed equipping the detector with the VFAT2 FEE [7] and it is showed in fig. 3. The $\sigma_{t}$ saturates at $5.7 \mathrm{~ns}$ due to the FEE. This value should be compared with the one measured by the LNF-LHCb-GEM group of $4.5 \mathrm{~ns}$ [9].

\section{Conclusions}

The $\mu$-RWELL technology is very promising and its R\&D is converging to a final resistive layout allowing to face up to $3 \mathrm{MHz} / \mathrm{cm}^{2}$ (for LHCb purposes) limiting the efficiency losses. The 


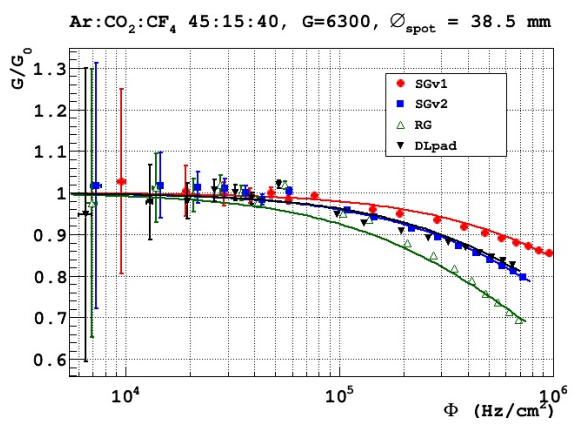

Figure 2: Rate capability comparison for the $\mu$-RWELL high rate versions.

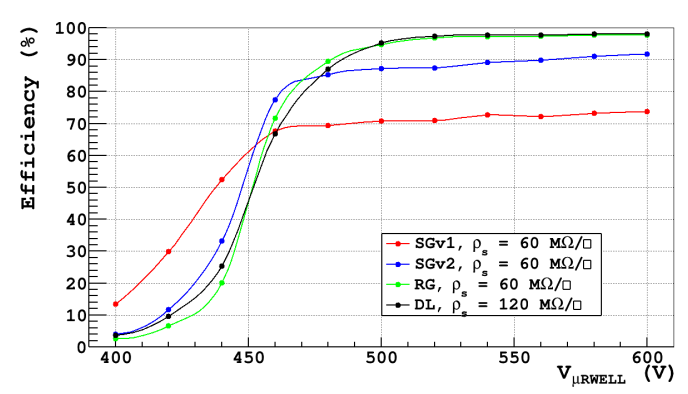

Figure 3: Tracking efficiency as function of the voltage applied to amplification stage for different $\mu$-RWELL layouts.

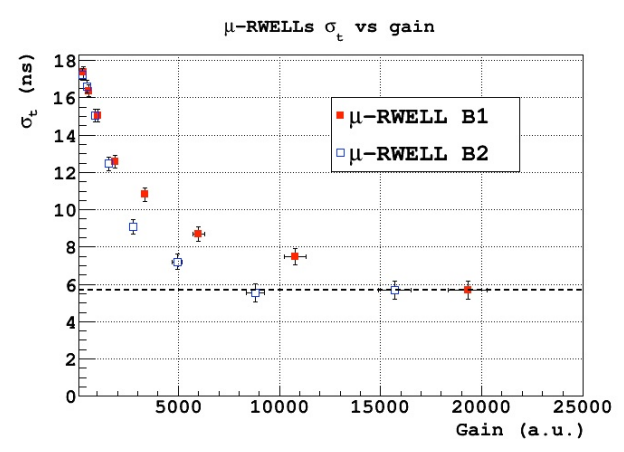

Figure 4: Time resolution as a function of the gain for a single resistive layer $\mu$-RWELL.

detector performance, measured with the LHCb-GEM gas mixture, are very interesting: a time resolution of 5.7 ns with a FEE saturation effect and a tracking efficiency higher than $92 \%$ with the SG2 detector. A new detector layout, based on the Single Layer layout and with a lower dead zone $(\sim 5 \%)$ has been produced and will be caracterized by the measurements with high intensity hadron beam. The cooperation with the companies (ELTOS, Be-Sputtering) is helping for a complete Technological Transfer of the detector technology.

\section{References}

[1] LHCb coll., CERN/LHCC 04 (1998)

[2] LHCb coll., CERN/LHCC 030 (2003)

[3] G. Bencivenni et al., JINST 10 (2015) P02008

[4] G. Bencivenni et al., NIM A 886 (2018) 36

[5] A. Alexoupulos et al., NIM A 617 (2010)

[6] L. Jones, https://cds.cern.ch/record/1069892/files/cer-002725643.pdf

[7] P. Aspell, https://cds.cern.ch/record/1069906/files/p292.pdf

[8] G. Bencivenni et al., PoS (BORMIO 2017) 002

[9] G. Bencivenni et al., NIM A 494 (2002) 156 